SLAC-PUB-8050

January 1999

\title{
Single Bunch Stability to Monopole Excitation*
}

\author{
Sam Heifets and Boris Podobedov \\ Stanford Linear Accelerator Center, Stanford University, Stanford, CA \\ 94309, USA
}

*Work supported by Department of Energy contract DE-AC03-76SF00515. 


\section{Abstract}

We study single bunch stability with respect to monopole longitudinal oscillations in electron storage rings. Our analysis is different from the standard approach based on the linearized Vlasov equation. Rather, we reduce the full nonlinear Fokker-Planck equation to a Schrödinger-like equation which is subsequently analyzed by perturbation theory. We show that the Haissinski solution [3] may become unstable with respect to monopole oscillations and derive a stability criterion in terms of the ring impedance. We then discuss this criterion and apply it to a broad band resonator impedance model.

\section{Introduction}

Single bunch longitudinal instability is one of the factors limiting the performance of electron storage rings. Theoretical analysis of this instability is usually based on the Fokker-Planck equation for the particle distribution function. This equation includes the effects of both dynamic (Hamiltonian) and stochastic forces. The Hamiltonian part describes the synchrotron motion while radiation terms account for the (much slower) effects of the synchrotron radiation and define the rms beam size at low intensity. Steadystate solution of the Fokker-Planck equation was first obtained in 1973 by J. Haissinski [3] and since then it was confirmed in numerous experiments held below the instability threshold. Unfortunately, apart from a few limiting cases, finding other possible solutions of the Fokker-Planck equation that could account for the instability turned out to be quite difficult. This is why much of the analysis to explain the instability was done utilizing the linearized Vlasov equation technique, where the Fokker-Plank equation was linearized with respect to the Haissinski solution. In this approach the 
Haissinski solution is also used to introduce the action-angle variables that make the Haissinski Hamiltonian independent of angle, which results in great simplification of further analysis.

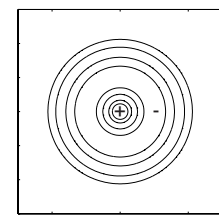

Monopole

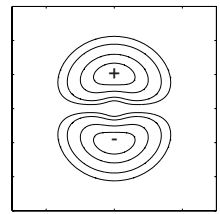

Dipole

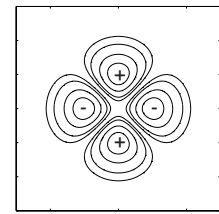

Quadrupole

Figure 1: Example contour plots of the lowest three azimuthal modes

The linearized Vlasov equation technique naturally leads to the concept of azimuthal phase space modes, that are basically the components of the perturbation to the Haissinski solution with certain azimuthal symmetry. The first three of such modes are sketched in Fig. 1. Note, that in this figure and throughout the rest of the paper we assume the simplest phase space topology, where action-angle variables can be defined uniformly across the whole plane. In other words, we neglect the possibility of several potential well minima.

As seen from Fig. 1 the monopole mode is quite special because, in contrast to other modes, its physical space projection does not change significantly on the time scale of a synchrotron period. This argues that radiation rather than Hamiltonian forces define the dynamics of this mode. Also, by definition of action-angle variables, the unperturbed Haissinski solution has monopole azimuthal structure. These two features of the monopole mode explain why it is omitted from the standard linearized Vlasov analysis. Indeed, in that approach the sole effect of radiation terms in the Fokker-Plank equation is that they define the Haissinski solution which subsequently cancels 
them out, so that only Hamiltonian terms remain in the linearized Vlasov equation. The possibility, that a perturbation is monopole, but with radial structure different from the Haissinski solution, is neglected.

In this paper we are exploring the possibility that an instability can be associated with the monopole mode. Rather than extending the linearized Vlasov technique we find it more convenient to transform the Fokker-Plank equation to a Schrödinger-like equation and then analyze the latter using the Haissinski solution as a basis. Advantages of this approach are that it is tractable and it conveniently allows us to use some well known facts about Schrödinger equation solutions.

The only essential approximation that we make in this paper is that we assume that the monopole mode can be considered separately form other azimuthal modes. This is, by no means, general. On the contrary, it is known that, for example, some collective instabilities result from azimuthal mode coupling, hence, concentrating on one mode in that case would be inappropriate. However, at lower intensity, when incoherent frequency shifts are small compared to the synchrotron frequency, the cross talk between different azimuthal modes is negligible. Whether a monopole mode or any other single azimuthal mode can become unstable at this low intensity is, in our opinion, a quantitative question that depends on the exact measure of the storage ring impedance. In fact, there are computer simulations for model impedances [2] showing that radial modes that belong to one azimuthal mode become unstable before any significant azimuthal mode coupling occurs. Of course, if a monopole mode does become unstable by itself, its independence from the other modes applies only to the initial stage of instability. The nonlinear stage may be rather complex and it falls beyond the scope of this 
paper.

Another argument to justify an independent consideration of the monopole mode refers to separate time-scales in this problem. Specifically, if we assume that the monopole mode instability develops slowly compared to the filamentation time, then the whole beam effectively maintains its initial monopole structure. Quantitatively, this amounts to a requirement that the incoherent frequency spread characteristic of the Haissinski equilibrium be much higher than the radiation damping rate. Such a condition is not unusual for many electron storage rings.

\section{Notation and basic equations}

For a relativistic bunch, $\gamma=E / m c^{2}>>1$, longitudinal dynamics is conveniently described in dimensionless variables

$$
x=z / \sigma_{0}, \quad p=-\delta / \delta_{0}, \quad \tilde{\tau}=\omega_{s 0} t
$$

where $z$ is position of a particle with respect to the bunch centroid $(z>0$ in the head of a bunch), $\delta$ is the relative energy spread $\Delta E / E, \omega_{s 0}$ is the synchrotron frequency. The subscript " 0 " refers to zero-current equilibrium quantities, related by $\omega_{0 s} \sigma_{0} / c=|\alpha| \delta_{0}$, where $\alpha$ is the momentum compaction. The Fokker-Planck equation for the distribution function $\rho(x, p, \tilde{\tau})$ in these variables can be written (e.g. [1]) as

$$
\frac{\partial \rho}{\partial \tilde{\tau}}+\{H, \rho\}_{p, x}=\frac{\gamma_{d}}{\omega_{s 0}} \frac{\partial}{\partial p}\left(\frac{\partial \rho}{\partial p}+p \rho\right)
$$

where $\{\ldots\}$ denotes the Poisson brackets, $H(x, p, \tilde{\tau})$ is the self-consistent Hamiltonian

$$
H(x, p, \tilde{\tau}) \equiv \frac{p^{2}}{2}+\frac{x^{2}}{2}+\Lambda \int d x^{\prime} d p^{\prime} \rho\left(x^{\prime}, p^{\prime}, \tilde{\tau}\right) S\left(x^{\prime}-x\right)
$$


and $\rho$ is normalized as $\int d p d x \rho(x, p, \tilde{\tau})=1$. We have neglected the nonlinearities of RF potential well and defined the parameter $\Lambda$ as

$$
\Lambda \equiv \frac{N r_{0}}{C \gamma \alpha \delta_{0}^{2}}
$$

where $N$ is number of particles in a bunch, $r_{0}$ is the classical electron radius, and $C$ is the ring circumference. We have also defined a dimensionless function

$$
S(x) \equiv \sigma_{0} \int_{0}^{x} d x^{\prime} W\left(\sigma_{0} x^{\prime}\right)
$$

in terms of the wakefield $W(z)$ for two particles separated by distance $z$. Causality was assumed in the form $W(z)=0$ for $z<0$.

The Fokker-Planck equation Eq. (2) has a steady-state Haissinski solution $[3]$

$$
\rho_{H}(x, p)=Z_{H} e^{-H_{H}(x, p)}
$$

where

$$
H_{H}(x, p)=\frac{p^{2}}{2}+\frac{x^{2}}{2}+\Lambda \int d x^{\prime} d p^{\prime} \rho_{H}\left(x^{\prime}, p^{\prime}\right) S\left(x^{\prime}-x\right)
$$

and $Z_{H}$ is a normalizing factor. Explicit forms of $\rho_{H}$ and $H_{H}$ can be obtained numerically.

Canonical transformation from $x, p$ to action-angle variables $J, \phi$ can be defined to make the Hamiltonian $H_{H}$ phase independent, $H_{H}(x, p) \rightarrow H_{H}(J)$.

Haissinski particle density $\rho_{H}$ in these variables depends only on $J$, and arbitrary distribution function $\rho(J, \phi, \tilde{\tau})=\rho_{H}(J)+\delta \rho(J, \phi, \tilde{\tau})$ can be expanded in azimuthal harmonics

$$
\rho(J, \phi, \tilde{\tau})=\rho_{H}(J)+\sum_{m=-\infty}^{\infty} \delta \rho_{m}(J, \tilde{\tau}) e^{i m \phi} .
$$


Similarly,

$$
\begin{aligned}
H(J, \phi, \tilde{\tau}) & =H_{H}(x, p)+\Lambda \int d x^{\prime} d p^{\prime} \delta \rho\left(x^{\prime}, p^{\prime}, \tilde{\tau}\right) S\left(x^{\prime}-x\right) \\
& =H_{H}(J)+\sum_{k=-\infty}^{\infty} \delta H_{k}(J, \tilde{\tau}) e^{i k \phi}
\end{aligned}
$$

where

$$
\delta H_{k}(J, \tilde{\tau}) \equiv \Lambda \sum_{m} \int d J^{\prime} S_{k, m}\left(J, J^{\prime}\right) \delta \rho_{k}\left(J^{\prime}, \tilde{\tau}\right)
$$

and

$$
S_{k, m}\left(J, J^{\prime}\right) \equiv \frac{1}{2 \pi} \int d \phi d \phi^{\prime} e^{i m \phi^{\prime}-i k \phi} S\left(x\left(J^{\prime}, \phi^{\prime}\right)-x(J, \phi)\right)
$$

In the $J, \phi$ variables the form of the left-hand-side of the Fokker-Planck equation is unchanged, appearing as in Eq. (2). The right-hand-side can be obtained using the invariance of the Poisson brackets [4]. Namely, for any $F \equiv F(x, p)$

$$
\frac{\partial F(x, p)}{\partial p}=\{x, F\}_{x, p}=\{x, F\}_{\phi, J} \equiv \frac{\partial}{\partial J}\left(\frac{\partial x}{\partial \phi} F\right)-\frac{\partial}{\partial \phi}\left(\frac{\partial x}{\partial J} F\right) .
$$

Hence, for the zero-th Fourier harmonic $\rho_{0}(J, \tilde{\tau}) \equiv\langle\rho(J, \phi, \tilde{\tau})\rangle$, where $\langle\ldots\rangle$ define phase averaging, we have

$$
\frac{\partial \rho_{0}}{\partial \tilde{\tau}}+\left\{H, \rho_{0}\right\}_{J, \phi}=\frac{\partial}{\partial J}\left(\frac{\partial x}{\partial \phi} \tilde{F}\right),
$$

where $\tilde{F} \equiv \partial \rho / \partial p+p \rho$. On the other hand, for any Hamiltonian $H=$ $p^{2} / 2+U(x, \tilde{\tau})$ the canonical momentum can be found as $p=\{x, H\}_{\phi, J}$. Therefore, if we neglect non-zero azimuthal modes by assuming $H=H(J, \tilde{\tau})$, $\rho=\rho_{0}(J, \tilde{\tau})$, we get

$$
\frac{\partial \rho}{\partial \tau}=\frac{\partial}{\partial J}\left[\left\langle\left(\frac{\partial x}{\partial \phi}\right)^{2}\right\rangle\left[\frac{\partial \rho}{\partial J}+\omega(J, \tau) \rho\right]\right] .
$$

where

$$
\omega(J, \tau) \equiv \frac{\partial H}{\partial J}=\omega_{H}(J)+\Lambda \int d J^{\prime} \delta \rho_{0}\left(J^{\prime}, \tau\right) \frac{\partial S_{0,0}}{\partial J} .
$$


As discussed earlier, the monopole mode should not change much on the time scale of a synchrotron period. This is why time was renormalized above to the radiation damping constant

$$
\tau \equiv \gamma_{d} t=\left(\gamma_{d} / \omega_{s 0}\right) \tilde{\tau}
$$

For the $x$ derivative in Eq. (14) we can write

$$
\left\langle\left(\frac{\partial x}{\partial \phi}\right)^{2}\right\rangle=\oint\left(\frac{\dot{x}}{\omega(J, \tau)}\right)^{2} d \tau \simeq \frac{1}{\omega(J, \tau)} \oint p d x \equiv \frac{J}{\omega(J, \tau)} \simeq \frac{J}{\omega_{H}(J)}
$$

where integration is performed over one synchrotron period and the last equality assumes small deviation from the Haissinski solution. Finally, introducing the diffusion coefficient as

$$
D(J) \equiv \frac{J}{\omega_{H}(J)},
$$

we can rewrite Eq. (14) as

$$
\frac{\partial \rho}{\partial \tau}=\frac{\partial}{\partial J}\left(D(J)\left[\frac{\partial \rho}{\partial J}+\omega(J, \tau) \rho\right]\right) .
$$

Note, that in our derivation we allow arbitrary time dependence of $H(J, \tau)$.

\section{Transformation to a Schrödinger-like equa- tion}

The Fokker-Planck equation Eq. (19) has a standard one-dimensional form that permits transformation to a Schrödinger-like equation [5]. Let us introduce a new independent variable

$$
y \equiv y(J)=\int_{0}^{J} d J^{\prime} / \sqrt{D\left(J^{\prime}\right)}
$$

and two functions

$$
f(y, \tau) \equiv \frac{1}{\sqrt{D(J(y))}} e^{\Phi(y, \tau) / 2} \rho(J(y), \tau),
$$




$$
\Phi(y, \tau) \equiv H(J(y), \tau)-(1 / 2) \ln D(J(y))
$$

where $J(y)$ in the right-hand-side of Eqs. (21-22) is given implicitly by Eq. (20). Now the Fokker-Planck equation Eq. (19) takes the form

$$
\frac{\partial f}{\partial \tau}=\frac{\partial^{2} f}{\partial y^{2}}-U_{S}(y, \tau) f+\frac{1}{2} \dot{\Phi}(y, \tau) f
$$

where

$$
U_{s}(y, \tau) \equiv\left[\Phi^{\prime}(y, \tau) / 2\right]^{2}-\Phi^{\prime \prime}(y, \tau) / 2
$$

and dot and prime denote partial derivatives with respect to $\tau$ and $y$ respectively. This equation is strongly nonlinear since, according to Eq. (15), $\Phi$ is also related to $f$ by

$$
\Phi(y, \tau)=\Lambda \int d y^{\prime} S_{0,0}\left(J, J^{\prime}\right)\left[e^{-\Phi\left(y^{\prime}, \tau\right) / 2} f\left(y^{\prime}, \tau\right)-e^{-\Phi_{H}\left(y^{\prime}\right) / 2} f_{H}\left(y^{\prime}\right)\right]
$$

where

$$
\begin{array}{r}
f_{H}(y) \equiv Z_{H} e^{-\Phi_{H}(y) / 2}, \\
\Phi_{H}(y) \equiv H_{H}(J(y))+\frac{1}{2} \ln \left(\frac{\omega_{H}(J(y))}{J(y)}\right), \\
\int d y e^{-\Phi_{H}(y) / 2} f_{H}(y)=1 .
\end{array}
$$

Note, that $f_{H}(y)$ is the steady-state solution of Eq. (23) and it corresponds to the Haissinski solution, as can be checked by direct substitution. However, it is not obvious how to proceed to other, time-dependent solutions of Eq. (23). On the other hand, if the $\dot{\Phi}$ term is neglected, the analysis of Eq. (23) can be made by analogy with more familiar quantum mechanical problems. Indeed, without that term Eq. (23) can be thought of as a Schrödinger equation for a particle in the potential well $U_{S}(y, \tau) .{ }^{\dagger}$ Whether the $\dot{\Phi}$ term

\footnotetext{
†Of course, the similarity is quite formal. The problem is purely classical, so no $\hbar$ appear anywhere. Also, there is no imaginary constant in front of the time derivative.
} 
is negligible for a general case remains unclear. However, since this term is zero for the Haissinski solution, one can safely neglect it for solutions that are close to $f_{H}$. This includes, for example, the important case of the early time behavior of a system initialized with the Haissinski distribution at $\tau=0$. In the next two sections we will follow this approach. Namely, we will first analyze Eq. (23) with the last term neglected, and then account for it by perturbation theory.

\section{Schrödinger equation analysis}

After neglecting the $\dot{\Phi}$ term Eq. (23) reads

$$
\frac{\partial f}{\partial \tau}=\frac{\partial^{2} f}{\partial y^{2}}-U(y, \tau) f
$$

First, we solve a linear problem for which $\omega(J)=\omega^{0}$ is a constant. In this case $y=2 \sqrt{\omega^{0} J}$ and the Schrödinger potential is simply

$$
U_{S}^{0}(y)=\frac{y^{2}}{16}-\frac{1}{2}-\frac{1}{4 y^{2}},
$$

which makes Eq. (27) a solvable eigenvalue problem. It is easy to check that the solution is

$$
f^{0}(y, \tau)=\sum_{m=0}^{\infty} \psi_{m}^{0}(y) e^{-\lambda_{m}^{0} \tau}
$$

where

$$
\begin{gathered}
\lambda_{m}^{0}=m, \quad m=0,1,2 \ldots, \\
\psi_{m}^{0}(y)=(y / 2)^{1 / 2} e^{-y^{2} / 8} L_{m}\left(y^{2} / 4\right),
\end{gathered}
$$

and $L_{m}$ denotes the Laguerre polynomial of order $m$. As expected, the linear problem does not have any unstable solutions. Any initial distribution expo-

nentially approaches the Haissinski solution $\psi_{0}^{0}(y)$ on the time-scale defined by radiation damping. 
For general case, $\omega(J) \neq$ const, asymptotic behavior of the solutions of Eq. (23) is described by the solutions to the linear problem Eqs. (28),(31). Namely, $f(y)$ scales as $\sqrt{y}$ at small $y$ and goes to zero as $P(y) e^{-y^{2} / 8}$ at large $y$, where $P(y)$ is a polynomial. Similarly, $U(y)$ is quadratic at large $y$ and has a $-1 / 4 y^{2}$ singularity as $y$ approaches zero.

Suppose that a bunch is described by the Haissinski distribution at $\tau=0$. By the foregoing arguments the behavior of this system for small $\tau$ can be obtained from Eq. (27). In this case

$$
f(y, \tau)=\sum_{m} \psi_{m}(y) e^{-\lambda_{m} \tau}
$$

where $\psi_{m}(y)$ are the eigenfunctions of the equation

$$
\frac{\partial^{2} \psi_{m}}{\partial y^{2}}-U_{S}(y, \tau) \psi_{m}=-\lambda_{m} \psi_{m} .
$$

Therefore, stability of the initial state depends on whether Eq. (33) has at least one negative eigenvalue $\lambda_{m}<0$. Let's look at the possibility that such negative eigenvalue exists.

First of all, the eigenfunctions with the asymptotic behavior described above are orthogonal and they can be normalized by

$$
\int \psi_{n}(y) \psi_{m}(y) d y=\delta_{n, m} .
$$

Hence, an eigenvalue of Eq. (33) is given by

$$
\lambda_{n}=-\int d y \psi_{n}\left[\frac{\partial^{2}}{\partial y^{2}}-U_{S}(y, \tau)\right] \psi_{n} .
$$

In spite of a singularity of $U_{S}(y, \tau)$ at $y=0$ all the eigenvalues of Eq. (33) are bounded from below. Indeed, at small distances where $\psi_{n} \propto \sqrt{y}$, the second derivative in Eq. (35) gives a $1 / 4 y^{2}$ term which cancels a similar term in $U_{S}(y)$. In fact, it can be shown that all the eigenvalues are higher than the average of the "effective potential" $V_{S}(y) \equiv U_{S}(y)+1 / 4 y^{2}$. 
As an example, we consider a broad-band resonator impedance model with shunt impedance $R_{s}$, resonance frequency $\omega_{R}$, and quality factor $Q$. The function $S(z)$ in this case is (e.g. [6])

$$
S(x)=\frac{I}{\Lambda Q \zeta} \sin (\sigma \zeta x) e^{-\sigma x / 2 Q},
$$

where we defined

$$
\begin{aligned}
I & \equiv 4 \pi \Lambda R_{s} / Z_{0}, \\
\sigma & \equiv \omega_{R} \sigma_{0} / c, \\
\zeta & \equiv \sqrt{1-1 /(2 Q)^{2}} .
\end{aligned}
$$

and $Z_{0}$ is the impedance of free space. The frequency $\omega_{H}(J)$ and the effective potential $V_{S}(y)$ for the parameters $Q=1$ and $\sigma=3$ are shown in Fig. 2 for two values of intensity $I=1$ and $I=-1$. Negative intensity corresponds to negative momentum compaction and the importance of this case will be discussed later. For these parameters the effective potential indeed has a minimum where $V_{S}(y)<0$. On the first sight, we could expect a mode trapped near the bottom of the potential well with the eigenvalue negative at large current. This, however, is not true since $\psi_{0}=f_{H}$ is the solution of Eq. (33) with $\lambda_{0}=0$. Because $f_{H}(y)$ does not have zeros, this solution has the lowest eigenvalue and the rest of $\lambda_{m}$ are positive.

Therefore, in this approximation, the Haissinski solution is stable. How much this conclusion depends on the assumption that the $\dot{\Phi}$ term in Eq. (23) is negligible can be analyzed by perturbation technique.

\section{Perturbation theory}

Eq. (23) and the condition of self-consistency Eq. (25) is a strongly nonlinear system of equations. We want to analyze it with perturbation theory, 

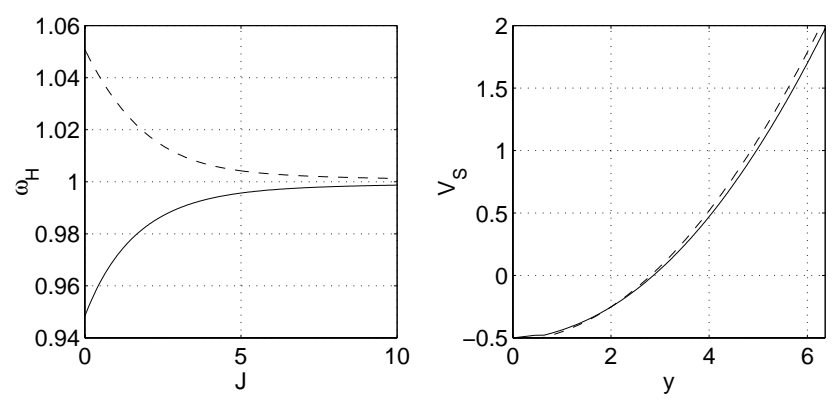

Figure 2: Incoherent frequency $\omega_{H}(J)$ and corresponding effective potential $V_{S}(y)$ (right) shown for broad band $Q=1$ resonator impedance with $\sigma=3$ and two values of intensity $I=1$ and $I=-1$ (dashed)

assuming small deviation from the Haissinski solution. Let's introduce perturbations as

$$
v(y, \tau) \equiv H(J(y), \tau)-H_{H}(J(y)), \quad \psi(y, \tau) \equiv f(y, \tau)-f_{H}(y, \tau)
$$

This gives $\Phi=\Phi_{H}(y)+v(y, \tau)$, and

$$
v(y, \tau)=\Lambda \int d y^{\prime} S_{0,0}\left(J(y), J\left(y^{\prime}\right)\right) e^{-\Phi_{H}\left(y^{\prime}\right) / 2}\left[\psi\left(y^{\prime}, \tau\right)-(1 / 2) v\left(y^{\prime}, \tau\right) f_{H}\left(y^{\prime}\right)\right]
$$

The perturbation $\psi(y, \tau)$ is normalized by the condition

$$
\int d y \psi(y, \tau) e^{-\Phi_{H}(y) / 2}=\frac{Z_{H}}{2} \int d y v(y, \tau) e^{-\Phi_{H}(y)},
$$

and satisfies the equation

$$
\frac{\partial \psi}{\partial \tau}=\frac{\partial^{2} \psi}{\partial y^{2}}-U_{H}(y) \psi+\frac{1}{2} f_{H}\left[\dot{v}+v^{\prime \prime}-\Phi_{H}^{\prime} v^{\prime}\right]
$$

Let's expand $\psi$ and $v$ in series over orthogonal eigenfunctions $\psi_{n}$

$$
\psi(y, \tau)=\sum_{m=0}^{\infty} C_{n}(\tau) \psi_{n}, \quad v(y, \tau)=\frac{2}{Z_{H}} \sum_{m=0}^{\infty} D_{n}(\tau) e^{\Phi_{H}(y) / 2} \psi_{n} .
$$


We assume that eigenfunctions $\psi_{n}$ satisfy the equation

$$
\psi_{n}^{\prime \prime}(y)-U_{H}(y) \psi_{n}(y)=-\lambda_{n} \psi_{n}(y) .
$$

The above expansion includes the eigenfunction $\psi_{0}(y)=\sqrt{Z_{H}} e^{-\Phi_{H}(y) / 2}$ with $\lambda_{0}=0$. Note, that by the same argument as above, the remaining eigenvalues are positive.

The linearized Fokker-Planck equation Eq. (43) and the condition of selfconsistency Eq. (41) lead to the following system

$$
\begin{gathered}
\dot{C}_{n}=\dot{D}_{n}-\lambda_{n}\left(C_{n}+D_{n}\right), \\
D_{n}=\Lambda \sum_{k} \kappa_{n, k}\left(C_{k}-D_{k}\right),
\end{gathered}
$$

where

$$
\kappa_{n, k} \equiv \int d y \psi_{n}(y) e^{-\Phi_{H}(y) / 2} \int d y^{\prime} \psi_{k}\left(y^{\prime}\right) e^{-\Phi_{H}\left(y^{\prime}\right) / 2} S_{0,0}\left(J(y), J\left(y^{\prime}\right)\right) .
$$

Due to orthogonality, the normalization condition Eq. (42) gives just

$$
C_{0}(\tau)=D_{0}(\tau)
$$

Since $\lambda_{0}=0$ this is automatically satisfied by Eq. (46) with the initial condition $C_{0}(0)=D_{0}(0)$.

Looking for exponentially varying solutions $C_{n} \equiv a_{n} e^{\mu \tau}, \quad D_{n} \equiv b_{n} e^{\mu \tau}$ we transform the system Eqs. (46)-(47) to the matrix equation

$$
b_{n}=-2 \Lambda \sum_{k} \kappa_{n, k} \frac{b_{k} \lambda_{k}}{\mu+\lambda_{k}} .
$$

The solution of this equation is given by the roots of the determinant for the matrix

$$
M \equiv \delta_{m, n}+2 \Lambda \frac{\kappa_{n, k} \lambda_{k}}{\mu+\lambda_{k}} .
$$


Positive roots $\mu>0$ would mean instability to monopole excitation of a bunch.

Since the matrix $M$ is infinite it is unclear how to find its determinant in the general case. However, it is easy to see, that off-diagonal terms of $\kappa_{n, k}$ are small, while the diagonal terms quickly converge to zero. This is why we expect that a good approximation for the roots $\mu$ can be found by truncating the matrix $M$. If we truncate matrix $M$ to the lowest nontrivial rank

$$
M^{(2)}=\left(\begin{array}{cc}
1 & 2 \Lambda \kappa_{1,1} \frac{\lambda_{1}}{\mu+\lambda_{1}} \\
0 & 1+2 \Lambda \kappa_{1,1} \frac{\lambda_{1}}{\mu+\lambda_{1}}
\end{array}\right),
$$

then zero determinant occurs for

$$
\mu=-\lambda_{1}\left(1+2 \Lambda \kappa_{1,1}\right)
$$

Because $\lambda_{1}>0$, this root is positive when

$$
2 \Lambda \kappa_{1,1}<-1
$$

and this may be viewed as the criterion for the onset of the monopole instability.

It is easy to see, however, that usually $\kappa_{1,1}>0$. Indeed, let's introduce impedance $Z(\nu)$ so that

$$
W(z)=\int \frac{d \nu}{2 \pi} Z(\nu) e^{-i \nu z / c}
$$

Then

$$
\kappa_{n, k}=i \frac{2 \sigma_{0}}{Z_{0}} \int \frac{d \nu}{2 \pi} \frac{Z(\nu)}{\nu} F_{n}(\nu) F_{k}^{*}(\nu)
$$

where

$$
F_{n}(\nu) \equiv \int d J\left(\frac{\omega_{H}(J)}{J}\right)^{1 / 4} e^{-H_{H}(J) / 2} \psi_{n}(y(J)) \int d \phi e^{i \nu \sigma_{0} x(J, \phi) / c}
$$


Now $\kappa_{1,1}$ depends on $\left|F_{1}(\nu)\right|^{2}$ which is positive and even function of $\nu$. Hence, $\kappa_{1,1}$ is given by the odd part of the impedance, $\operatorname{Im} Z(\nu)$ which is negative for inductive impedance and positive for capacitive impedance. As a result, for the most common case of inductive impedance and positive momentum compaction, $\Lambda \kappa_{1,1}>0$ and the Haissinski solution is stable.

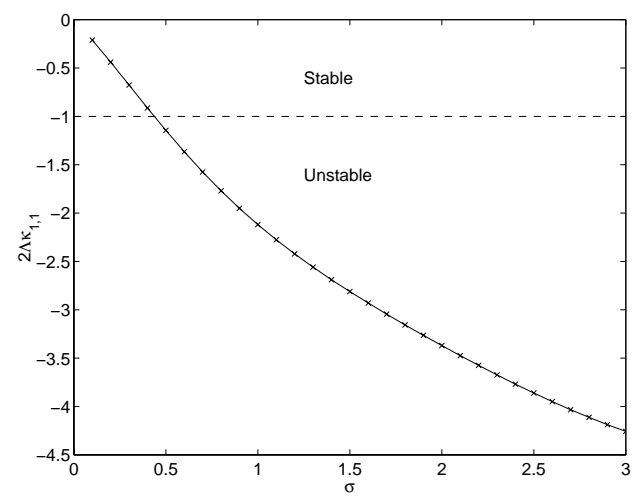

Figure 3: Illustration for the monopole instability criterion Eq. (54) for broad band $(\mathrm{Q}=1)$ resonator impedance for negative momentum compaction and intensity $I=-1$. Numerical calculation was done using Eqs. (56)-(57) where $\omega(J)$ was assumed constant and $\psi_{1}$ was taken from the solution to the linear problem Eq. (31).

However, the situation is not that simple for negative momentum compaction or in the case of capacitive impedance. Each of these conditions have been proposed by various authors to get shorter bunches and also as a remedy against longitudinal instabilities. For illustration, we continue our example of the broad band resonator model for $Q=1$. Using Eqs. (56)-(57) we numerically compute the quantity $2 \Lambda \kappa_{1,1}$ as function of $\sigma$ at intensity $I=-1$. The result together with the threshold value given by Eq. (54) is plotted in Fig. 3. It shows that a bunch is monopole unstable at this intensity provided its zero current length exceeds about one twelveth of the resonator wavelength. Note, that according to Fig. 2 this intensity is not 
high at all since, for example, for $\sigma=3$, it only leads to about $5 \%$ increase in the incoherent frequency spread.

\section{Discussion}

We have investigated single bunch stability with respect to longitudinal monopole oscillations. These oscillations may become unstable as a result of an imbalance between radiation excitation and damping. Since this phenomenon falls beyond the coverage of the linearized Vlasov approach, we chose to employ a different technique that has not been used for instability analysis. This technique involves the transformation of the phase-averaged Fokker-Planck equation to a Schrödinger equation with an additional term arising from the self-consistent potential. This Schrödinger equation is analyzed similar to quantum mechanics and the effects of the additional term are found by perturbation analysis.

Utilizing this technique we have obtained a simple criterion, Eq. (54) for the onset of monopole instability. According to this criterion, monopole mode instability does not appear in the most common case of storage ring operation with positive momentum compaction when the impedance is largely inductive. However, for the case of negative momentum compaction $\alpha<0$, as we have illustrated in Fig. 3, bunches may become monopole unstable at modest intensity. We expect a similar behavior for the somewhat rare case of predominantly capacitive impedance and $\alpha>0$.

As discussed in the introduction, the essential assumption we make in our analysis is that the monopole mode can be considered separately from the rest of azimuthal modes. This assumes the absence of azimuthal mode coupling which implies some limitations on the growth rate of the instability. 
It also assumes that the other azimuthal modes are stable by themselves. It is interesting, that since the monopole instability criterion Eq. (54) effectively includes only the imaginary part of impedance, the second assumption is rather relaxed. Indeed, as follows from the linearized Vlasov analysis, the azimuthal modes other than monopole, become unstable due to the asymmetry in the Haissinski potential that comes from the real part of impedance (We omit a somewhat exotic possibility of multiple minima in the Haissinski potential). Therefore, monopole mode instability can exist when the remaining azimuthal modes are stable.

It is conceivable, that the monopole instability could be one of the factors that prevent high current operation of storage rings with negative momentum compaction. Many attempts of such operation have been tried to shorten a bunch and to avoid various instabilities (e.g. [7], [8]), often the so-called microwave instability(e.g. [6]). Unfortunately, since, usually only the static bunch shape and/or the energy spread measurements are reported, it is hard to infer what particular instability was the limitation. However, in some cases, it appears that there is something other than the microwave instability, because the threshold reduction predicted for this instability (e.g. [9]) is not observed. It would be nice to find a concrete evidence of monopole instability in either future experiments or in the log books from the past ones. Such evidence might include, for example, growth of the longitudinal beam size, in the absence of synchrotron sidebands to the revolution harmonics of a beam position monitor signal.

Finally, we hope that the technique described in this paper can be applied to other problems in accelerator physics that lead to the one dimensional Fokker-Planck equation. This include, for example, microwave instability, 
beam-beam interaction in collider rings, and even halo formation in rings and linacs. It would be especially interesting if, for any of these problems, along with a steady state solution with $\lambda=0$, there exists an exponentially growing solution of the Schrödinger equation that has a negative eigenvalue $\lambda<0$. This could qualitatively explain the relaxation oscillation behavior seen in many numerical and real life experiments.

\section{Acknowledgments}

We thank Alex Chao and Bob Siemann for insightful discussions and support of this study, and also Albert Hoffman for pointing out the relevance of the negative momentum compaction case to this problem. Many thanks are also due to Dave Whittum for reading this manuscript and providing valuable comments. This work was supported by the Department of Energy Contract No. DE-AC03-76SF00515. 


\section{References}

[1] H. Bruck, in Accelerateurs circulaires des particles, (Presses Universitaires de France, Paris, 1966).

[2] K. Oide, KEK Preprint 94-138, (1994).

[3] J. Haissinski, Nuovo Cimento 18B, 72, (1973).

[4] J. Schonfeld, Ann. Phys. 160, 149, (1985).

[5] H. Risken, in The Fokker-Planck Equation, (Springer, Berlin, 1988).

[6] A. Chao, Physics of Collective Beam Instabilities in High Energy Accelerators, (J. Wiley \& Sons, Inc, New York, 1988).

[7] M. Hosaka et. al., Nuclear Instr. and Meth. in Physics Research A 407 (1998) 234-240.

[8] A Nadji et. al., Proc. EPAC-96, Barcelona, 676, (1996).

[9] S.X. Fong et. al., KEK Preprint 94-190, (1994). 